\title{
Drug abuse and its ramifications on skeletal system
}

\author{
Das Sanjita* and Kumar Naveen \\ Noida Institute of Engineering and Technology (Pharmacy Institute), India
}

\section{Abstract}

The purpose of this study is to highlight the drug abuse hazards and preventive aspects. From a public health perspective, substance abuse has long been a source of major concern, both for the individual's health and for wider society as a whole. The UK has the highest rates of recorded illegal drug misuse in the western world. In particular, it has comparatively high rates of heroin and crack cocaine use. Substances that are considered harmful are strictly regulated according to a classification system that takes into account the harms and risks of taking each drug. The adverse effects of drug abuse can be thought of in three parts that together determine the overall harm in taking it. Some addictive substances are more damaging to the skeletal system along with the others. In this review article, an effort has been taken to elaborate the effects of addictive drugs on human highlighting these most problematic substances for bones and also the promising potential prevention aspects of drug abuse.

\author{
More Information \\ *Address for Correspondence: Das Sanjita, \\ Noida Institute of Engineering and Technology \\ (Pharmacy Institute), Plot No.19, Knowledge \\ Park-Il, Greater Noida-201306, Uttar Pradesh, \\ India, Tel: 9818223359; \\ Email: sanjita8@yahoo.co.in
}

Submitted: 25 October 2019

Approved: 04 November 2019

Published: 05 November 2019

How to cite this article: Sanjita D, Naveen K. Drug abuse and its ramifications on skeletal system. Int J Bone Marrow Res. 2019; 2: 083-086.

DOI: dx.doi.org/10.29328/journal.ijbmr.1001008

Copyright: (c) 2019 Sanjita D, et al. This is an open access article distributed under the Creative Commons Attribution License, which permits unrestricted use, distribution, and reproduction in any medium, provided the original work is properly cited.

Keywords: Drug abuse; Addiction; Bones; Skeletal system; Arthritis

\section{Check for updates}

\section{Introduction}

There's a fine line between regular drug use and drug abuse and addiction. Very few drug abusers or addicts are able to recognize when they've crossed that line. While frequency or the amount of drugs consumed do not necessarily constitute drug abuse or addiction, they can often be indicators of drug-related problems. Drugs are chemicals that disrupt the brain the nerve cell function. The Major substance causing drug addiction are marijuana, hashish and other cannabis-containing substances, $\mathrm{K} 2$, spice and bath salts, barbiturates, benzodiazepines and hypnotics, methamphetamine, cocaine and other stimulants, hallucinogens, inhalants, opioid painkillers, etc. Addiction is all about getting accustomed to a particular habit which itself is a complex disease and in order to quitting this habit depends upon an individual's strong will power [1].

Drug abuse is a patterned use of a drug in which the user consumes the substance in amounts or with methods which are harmful to themselves or others, and is a form of substancerelated disorder. Widely differing definitions of drug abuse are used in public health, medical and criminal justice contexts. In some cases criminal or anti-social behaviour occurs when the person is under the influence of a drug, and long term personality changes in individuals may occur as well. In addition to possible physical, social, and psychological harm, use of some drugs may also lead to criminal penalties, although these vary widely depending on the local jurisdiction [2]. There have been numerous research studies that have suggested that the density of an individual's bones can be affected by chronic substance abuse. In the present study the dangerous effect of drug abuse on skeletal system is highlighted along with its effect on other systems and their prevention.

\section{Causes of drug abuse}

Drug addiction can start with experimental use of a recreational drug in social situations, and, for some people, the drug use becomes more frequent. For others, particularly with opioids, drug addiction begins with exposure to prescribed medications, or receiving medications from a friend or relative who has been prescribed the medication.

The risk of addiction and how fast you become addicted varies by drug. Some drugs, such as opioid painkillers, have a higher risk and cause addiction more quickly than others. As time passes, you may need larger doses of the drug to get high. Soon you may need the drug just to feel good. As your drug use increases, you may find that it's increasingly difficult to go without the drug. Attempts to stop drug use may cause intense cravings and make you feel physically ill (withdrawal symptoms) [3].

\section{Signs that abuse has become addiction}

Substance abuse can lead to substance dependence or addiction when both the amount of substance used and the rate of use increase. People who experience drug or alcohol addiction, for example, occurs when people become 
chemically dependent on alcohol and those who are addicted may become ill if they suddenly stop drinking. People may also feel psychologically dependent on a substance.

- Feeling that you have to use the drug regularly - daily or even several times a day

- Having intense urges for the drug that block out any other thoughts

- Over time, needing more of the drug to get the same effect

- Taking larger amounts of the drug over a longer period of time than you intended

- Making certain that you maintain a supply of the drug

- Spending money on the drug, even though you can't afford it

- Not meeting obligations and work responsibilities, or cutting back on social or recreational activities because of drug use

- Continuing to use the drug, even though you know it's causing problems in your life or causing you physical or psychological harm [4].

\section{Effect of drug abuse on skeletal system}

There are several types of conditions that may be exacerbated by a history of substance abuse and, in some cases, may be caused by chronic substance abuse; again, causeand-effect relationships are not well demonstrated in research studies. Research has demonstrated that individuals with chronic substance abuse issues (e.g., methamphetamine abuse) as a group have lower bone densities than individuals who have no history of substance abuse or diagnosis of a substance use disorder. The conditions include osteoporosis, osteomyelitis, tooth decay, spinal conditions, and different forms of arthritis, leading to increased bone fragility and susceptibility to fracture. Primary osteoporosis refers to a reduction in bone mass related to aging and menopause, whereas secondary osteoporosis results from specific diseases or drugs. Druginduced osteoporosis typically refers to bone loss resulting from a variety of drugs, most notably oral glucocorticoids, antidepressants, antipsychotics, anticonvulsants, gastric acidreducing agents, and thyroid drugs. Alcohol consumption, especially excessive alcohol consumption associated with alcoholism, can increase a person's likelihood of developing osteoporosis as they age. When you consume too much alcohol the stomach finds it increasingly difficult to absorb calcium and the necessary minerals it needs to function properly, resulting in deficiencies of vital components for healthy bones.

- Alcohol: People who struggle with alcohol use disorder disrupt the ability of their digestive system to absorb calcium, and on a long-term basis, this can lead to osteoporosis, osteoarthritis, or other brittle bone conditions. This increases the risk of fractures or breaks, especially when combined with the loss of physical coordination that occurs when a person is drunk. Alcohol and drug consumption also inhibits the pancreas' ability to absorb calcium and vitamin D. In women, alcohol and drug use decreases estrogen levels. Estrogen is vital for the remodeling of bones and slows down the natural process of bone loss. Alcohol isn't just dangerous to bones; studies are showing an increased link between alcoholism and dementia.

- Benzodiazepines: These prescription psychiatric medications are often abused, as they induce a sense of relaxation and euphoria rapidly after a person takes them. That intoxication causes side effects like muscle weakness and pain, as well as a loss of physical coordination that looks like the person is drunk. This can lead to falls, fractures, sprains, and other problems with the musculoskeletal system.

- Inhalants: This type of substance abuse is extremely dangerous and likely to cause damage to some body systems upon first use. Chronic abuse of inhalants like benzene damages bone marrow. Other inhalants cause skeletal abnormalities if abused over a long time.

- MDMA: Also called Molly and sometimes confused with ecstasy, this amphetamine is a club drug that can lead to serious consequences. One of the most dangerous effects for the musculoskeletal system is hyperthermia, which leads to muscle breakdown similar to rhabdomyolysis. This can poison other organ systems, especially the kidneys. The temporomandibular joint (TMJ) acts like a sliding hinge, connecting your jawbone to your skull. You have one joint on each side of your jaw. TMJ and tooth decay are often due to habitual teeth-grinding and clenching as it's associated to stimulation and stress. Smoking methamphetamine, MDMA, and crack are also known to affect the saliva glands, reducing protection from tooth decay and erosion. Some common side effects include trouble eating or swallowing, difficulty talking, and soreness. In most cases, the pain and discomfort associated with TMJ disorders is temporary and can be relieved with self-managed care or nonsurgical treatments.

- Opioids: Prescription versions of these drugs are often prescribed to alleviate muscle and bone pain from many conditions, ranging from fractures or surgery to chronic illnesses like arthritis or cancer. However, opioid drugs can cause side effects like muscle aches and pain. Additionally, opioids like heroin, which are shot directly into the veins, can cause infections of the tendons or joints (osteomyelitis or septic arthritis). Brown heroin, in particular, can lead to side effects like joint stiffness, muscle pain and aches, and low back pain. 
- Steroids: When adolescents or young adults abuse steroids, the drug forces bones to stop growing, leading to shorter stature and potential bone problems later in life. Abuse of steroids can also change the ability of muscles to grow and cause tendon rupture in any age group. The practice of doping in athletes adds stress to muscles and bones, increasing the risk of fractures and torn muscles. Steroid injections are typically done into muscle tissue, which can cause muscle infections.

- Cocaine: It may affect the bones in some users by reducing bone density, which is often a side effect of the drug's effects on blood cells, appetite, and overall absorption of nutrients. Prolonged cocaine use has been linked to peripheral erythrocytosis in bone marrow along with a condition in which the body has an excess concentration of red blood cells within the veins and causes symptoms such as shortness of breath, nosebleeds, itching, and increased blood pressure $[5,6]$.

However, an equally serious threat comes from nicotine, alcohol, and other commonly abused drugs, such as opioids. Opium consumption is usually accompanied by specific risky life styles such as cigarette smoking, low physical activity and poor nutrition. Poor nutritional status among them who are addicted to opium is a result of economical problems and loss of appetite. Opium addiction can lead to low dietary intake of calcium and phosphor and indirectly makes the individual more prone to bone loss. Opium addicted patients are more susceptible to bone loss than non-addicted individuals. So, early screening and conducting prevention programs should be taken into consideration for this high risk group [7].

In another study it has been studied the possible consequences of chronic opiate abuse on bone mass, bone turnover and calcium metabolism in 13 male chronic heroin users. The chronic abuse of opioid drugs may be associated with altered bone metabolism and reduced trabecular bone mass, attributable, at least in part, to gonadal deficiency. These alterations seem reversible after drug discontinuation [8]. Lifetime substance abuse or dependence appears to have an adverse association with survival after bone marrow transplant when other clinical factors are equal [9].

\section{Prevention and treatment of drug addiction}

- Stick with your treatment plan: Monitor your cravings. It may seem like you've recovered and you don't need to keep taking steps to stay drug-free. But your chances of staying drug-free will be much higher if you continue seeing your therapist or counselor, going to support group meetings and taking prescribed medication.

- Avoid high-risk situations: Don't go back to the neighborhood where you used to get your drugs. And stay away from your old drug crowd.
- Get help immediately if you use the drug again: If you start using the drug again, talk to your doctor, your mental health professional or someone else who can help you right away [10].

Current addiction treatments use a combination of counseling and complete abstinence, slow weaning, or drug replacement that either substitutes for the drug or blocks withdrawal symptoms. Although these therapies control physical cravings, they don't seem to reverse the lasting changes in the brain caused by drug abuse, and therefore may only provide a temporary fix. Cognitive behavioral therapy and motivational interviewing therapy: A person centered therapy that relies on the person's inspiration to change has demonstrated effectiveness in this arena. Sometimes therapy provides a supplemental form of support for someone who is attending a self-help group [11]. Opium addiction can lead to low dietary intake of calcium and phosphor and indirectly makes the individual more prone to bone loss. When individuals experience wear and tear in their joints, attention towards proper nutrition rich in minerals like calcium is recommended to counteract the development of osteoarthritis along with the general preventions measures for drug abuse. Rheumatoid arthritis, which is believed to be due to an autoimmune disease, may be more common in individuals with chronic substance abuse issues. As in many of these arthritic disorders, the joints become deformed and may need to be replaced; hence the symptomatic treatment and targeted treatment are required. Animal studies showed promising results after hippocampal transplantation of BM-MSCs in psychological disorders e.g. depression and alcohol abuse [12].

\section{Conclusion}

Talk therapies, such as cognitive behavioral therapy (CBT), are usually what people think of when they consider what rehab might entail. Both individual and group sessions are important in the rehab setting and can help clients on their recovery journeys. In case of inhalant Practitioners should maintain a high index of suspicion for inhalant use, screen for inhalant use and inhalant use disorders, and intervene early in the course of the disorder with educational interventions and approaches that have been used in the treatment of other substance use disorders (e.g., motivational enhancement and relapse prevention interventions). This approach seems reasonable until researchers develop and fully evaluate effective evidence-based interventions for inhalant abusers. From the present study it has been concluded that addicted patients were more susceptible to bone loss than nonaddicted individuals. So, early screening and conducting prevention programs should be taken into consideration for this high risk group. The present study suggests that drug abused patients are more susceptible to bone loss than nonaddicted individuals. In addition to indirect effects of opium, this substance may also contribute to lowered bone mineral density and increased fracture risk by directly interfering 
with bone formation. Inhibition of human osteoblastic tissue cultures growth, decrease in serum osteocalcin levels and finally inhibition of osteocalcin production by osteoblast tissue cultures are some direct effects of opium on bone formation. In scientific studies it has also been reported that an increased level of prolactin in concurrent opium and cigarette smokers in comparison with cigarette smokers alone (86.96\% vs. $41.65 \%$ ) [13]. Other researches also reached the same results [13]. Hyperprolactinemia is one of the well known risk factors for developing bone loss that is another explanation for increased risk of bone loss and bone fracture in opium addicted persons. So, early screening and conducting prevention programs should be taken into consideration for this high risk group. There are some researches about the possible mechanisms of bone loss by drug abuse. Without any doubt, further studies to explore responsible mechanisms are needed. It may help for further study on drug abuse to reduce its occurrence. It may also be helpful for further research in the treatment and prevention of drug abuse mainly its harmful effects on skeletal system.

\section{References}

1. Nutt D, King LA, Saulsbury W, Blakemore C. Development of a rational scale to assess the harm of drugs of potential misuse. The Lancet. 2007; 369: 1047-1053.

PubMed: https://www.ncbi.nlm.nih.gov/pubmed/17382831

2. Mosby's Medical, Nursing \& Allied Health Dictionary. Drug abuse definition. 2002; 552.

3. Fonseca $\mathrm{F}$, Gilchrist $\mathrm{G}$, Torrens $\mathrm{M}$. Integrating addiction and mental health networks to improve access to treatment for people with alcohol and drug-related problems: A qualitative study. Adv Dual Diagn. 2012; 5: 5-14.
4. National Institute on Drug Abuse (NIDA). NIH. NIDA Info Facts. 2019.

5. Weber JE, Larkin GL, Boe CT, Fras A, Kalaria AS, et al. Effect of cocaine use on bone marrow-mediated erythropoiesis. Acad Emerg Med. 2003; 10: 705-708.

PubMed: https://www.ncbi.nlm.nih.gov/pubmed/12837643

6. Gozashti MH, Shahesmaeili A, Amini Zadeh Iran N. Is Opium Addiction a Risk Factor for Bone Loss? Red Crescent Med J. 2011; 13: 464-468.

7. Pedrazzoni $M$, Vescovi PP, Maninetti L, Michelini $M$, Zaniboni $G$, et al Effects of chronic heroin abuse on bone and mineral metabolism. Acta Endocrinol (Copenh). 1993; 129: 42-45.

PubMed: https://www.ncbi.nlm.nih.gov/pubmed/8351958

8. Chang G, Antin JH, Orav EJ, Randall U, McGarigle C, et al. Substance abuse and bone marrow transplant. Am J Drug Alcohol Abuse. 1997; 23: 301-308. PubMed: https://www.ncbi.nlm.nih.gov/pubmed/9143640

9. Wilkinson ST, Radhakrishnan R, D'Souza DC. Impact of Cannabis Use on the Development of Psychotic Disorders. Curr Addict Rep. 2014; 1: 115-128. PubMed: https://www.ncbi.nlm.nih.gov/pubmed/25767748

10. Stall R, Paul JP, Greenwood G, Pollack LM, Bein E, et al. Alcohol use, drug use and alcohol-related problems among men who have sex with men: the Urban Men's Health Study. Addiction. 2001; 96: 1589-1601. PubMed: https://www.ncbi.nlm.nih.gov/pubmed/11784456

11. Balster RL. Inhalant abuse, a forgotten drug abuse problem; College on Problems of Drug Dependence Presidential Address. 1996.

12. Rafaiee R, Ahmadiankia N. Bone Marrow Derived Mesenchymal Stem Cells in Addiction Related Hippocampal Damages. Int J Mol Cell Med. 2018; 7: 70-79.

PubMed: https://www.ncbi.nlm.nih.gov/pubmed/30276162

13. Ellingboe J, Mendelson $\mathrm{JH}$, Kuehnle JC. Effect of heroin naltrexone on plasma prolactin levels in man. Pharmacol Biochem Behav. 1980; 12 163-165.

PubMed: https://www.ncbi.nlm.nih.gov/pubmed/7367458 